Article

\title{
Regional Disparities in the Association between Cereal Consumption and Metabolic Syndrome: Results from the China Health and Nutrition Survey
}

\author{
Lina Huang ${ }^{\circledR}$, Huijun Wang, Zhihong Wang, Jiguo Zhang $®$, Bing Zhang and Gangqiang Ding * \\ National Institute for Nutrition and Health, Chinese Center for Disease Control and Prevention, \\ Beijing 100050, China; huangln2999@126.com (L.H.); wanghj@ninh.chinacdc.cn (H.W.); \\ wangzh@ninh.chinacdc.cn (Z.W.); zhangjg@ninh.chinacdc.cn (J.Z.); zhangbing@chinacdc.cn (B.Z.) \\ * Correspondence: dinggq@chinacdc.cn; Tel.: +86-10-6623-7001
}

Received: 15 January 2019; Accepted: 28 March 2019; Published: 1 April 2019

check for updates

\begin{abstract}
This study examines regional disparities in the association between cereal consumption and metabolic syndrome (MetS) among Chinese adults. We used data from the longitudinal China Health and Nutrition Survey (CHNS) for 2892 healthy adults aged 18-75 years (1088 in northern China, 1804 in southern China) who had no non-communicable chronic diseases or MetS at the initial visit in 2009 and the follow-up in 2015. We used a 74-item food frequency questionnaire (FFQ) to assess the dietary intake. We defined MetS according to the International Diabetes Federation (IDF) criteria. Multiple logistic regressions stratified by region were performed to estimate the association between cereal consumption and the risk of MetS, and the quantile regression analyzed the relationship between cereal consumption and individual components of MetS in 2015. The rice consumption in southern China (9.00 kg/month) was more than twice that in northern China $(3.60 \mathrm{~kg} / \mathrm{month})$. Consumption of wheat and wheat products in northern China (4.20 kg/month) was more than twice that in southern China $(1.50 \mathrm{~kg} / \mathrm{month})$. After we adjusted for potential confounders, rice consumption was inversely associated with a risk of MetS 0.709 (95\% CI: 0.458-1.003), the intake of wheat and wheat products was positively associated with a risk of MetS 1.925 (95\% CI: 1.292-2.867) in southern China. We found no association between the intake of cereal and the prevalence of MetS in northern China. The quantile regression showed that various cereals were differentially associated with the components of MetS. The association between cereal consumption and the risk of MetS, and the components of MetS varied across these two regions of China.
\end{abstract}

Keywords: metabolic syndrome; regional disparity; cereals

\section{Introduction}

Cardiovascular diseases (CVDs) are the leading cause of disease burdens and deaths globally [1,2]. In 2015, an estimated 290 million people had CVDs, and it is also the number one cause of mortality in China, accounting for more than $40 \%$ of all deaths [3]. The Metabolic syndrome (MetS) is a cluster of metabolic abnormalities that have been associated with an increased risk of developing CVDs and type 2 diabetes mellitus (T2DM) [4,5], along with central obesity, increased blood pressure (BP), increased fasting plasma glucose (FPG), increased triglycerides (TG), and decreased high-density lipoprotein cholesterol (HDL-C). The prevalence of MetS has increased dramatically and has become a serious public health problem in China and worldwide. Based on the findings from the China National Health and Nutrition Surveillance (2010-2012), the overall prevalence rate of MetS among Chinese adults was 11.0\% [6].

Various studies have focused on the relationship between cereal consumption and the prevalence of MetS. The literature on rice intake is mixed, with some studies finding a positive relationship $[7,8]$, some no relationship [9-13], and some an inverse relationship [14,15]. In the Japanese population, 
whole-wheat consumption has been shown to significantly reduce visceral fat obesity, and therefore reduce the risk of MetS [16], but udon noodle consumption was shown to increase the risk of MetS and abdominal obesity [15]. That study also reported that the intake of wheat as a standard high-fiber food might improve the cardiovascular risk profile [17]. Coarse cereals including maize, lentils and legumes, and minor millets, are rich in compounds that help prevent several chronic diseases like CVDs, T2DM, and obesity $[18,19]$. Several cross-sectional studies among Chinese populations have found different results on the relationship between tuber consumption and the prevalence of MetS [20,21]. In China, rice and wheat are the main staple foods, with regional variations (rice consumption is higher in southern China than in northern China, and wheat consumption is higher in northern China than in southern China). Although cereal consumption in China has decreased over the past three decades, nearly $53.1 \%$ of the total caloric intake is still derived from cereals [22]. However, few studies have compared the regional disparities in cereal consumption and the prevalence of MetS in the two regions, especially the prospective study. We used the China Health and Nutrition Survey (CHNS) data to examine the associations between the consumption of individual staple foods and the prevalence of MetS, with attention to regional variations.

\section{Materials and Methods}

\subsection{Study Design and Subjects}

All data used in this study are from the CHNS, an ongoing large-scale, longitudinal, household-based survey of ten waves (1989-2015). The CHNS originally covered nine provinces in 1989 that varied in demography, geography, economic development, and public resources. It used a multistage, stratified, random cluster process to select communities for the sample. In each community, 20 households were randomly selected and all individuals were surveyed for all the data in each wave. The survey procedure has been described in detail elsewhere [23]. Based on the natural boundary of a line along the Qinling Mountains and the Huaihe River, China is divided into northern and southern geographic regions [24]. The nine provinces selected in the original CHNS are disturbed in these two regions, Jiangsu, Hubei, Hunan, Guangxi and Guizhou in southern China; Heilongiiang, Liaoning, Shandong and Henan in northern China. The survey has only collected fasting blood samples in 2009 and 2015; so, our analysis considered 5132 adults aged 18-75 years who participated in those two waves. Of those participants, we excluded people diagnosed with hypertension, diabetes, cancer or who had MetS in the 2009 wave; pregnant and lactating women; and people with missing diet, BP, waist circumference (WC), or fasting blood data in 2009 or 2015. Hence, our final sample included 2892 people (1088 from northern China, 1804 from southern China). And 728 participants ( 354 from northern China, 374 from southern China) developed into MetS in 2015.

All participants gave their written informed consent before participating in the study. The study was approved by the Institutional Review Board of the University of North Carolina at Chapel Hill and the National Institute for Nutrition and Health, Chinese Center for Disease Control and Prevention (2015017).

\subsection{Dietary Data}

Our dietary assessment used a semi-quantitative food frequency questionnaire (FFQ) during the baseline visit. The FFQ included 74 food items and nine food categories: cereals, including rice, wheat and wheat products, other staple cereal and tuber; legumes; vegetables; fruits; dairy products; meats, including pork, beef, mutton, poultry, fish and shrimp; eggs; snacks; and alcohol and soft drinks [25]. For each food item, participants reported the frequency of habitual consumption (daily, weekly, monthly, annually or never (the reference category)) and the amount consumed over the past 12 months. Staple foods (e.g., rice, wheat) are consumed daily, but for the episodically consumed (e.g., coarse cereals and tuber) we used monthly consumptions. We converted individual consumption of food items to kilograms per month in the analysis. It was not possible to accurately calculate total energy intake (TEI) from the FFQ, so we used three consecutive 24-h dietary recalls to calculate the TEI. 


\subsection{Definition of MetS}

The definition of MetS was based on recommendations from the International Diabetes Federation (IDF) and AHA/NHLBI criteria [26]. According to this definition, a person diagnosed with MetS must meet at least three out of five criteria: (1) Elevated WC (using the Chinese values: $\geq 90 \mathrm{~cm}$ in man, $\geq 80 \mathrm{~cm}$ in female); (2) raised TG (TG $\geq 150 \mathrm{mg} / \mathrm{dL}(1.7 \mathrm{mmol} / \mathrm{L})$ or drug treatment for elevated triglycerides); (3) reduced HDL-C $(<40 \mathrm{mg} / \mathrm{dL}(1.0 \mathrm{mmol} / \mathrm{L})$ in males, $<50 \mathrm{mg} / \mathrm{dL}(1.3 \mathrm{mmol} / \mathrm{L})$ in females or drug treatment for reduced HDL-C); (4) raised BP (systolic BP [SBP] $\geq 130 \mathrm{mmHg}$ or diastolic $\mathrm{BP}[\mathrm{DBP}] \geq 85 \mathrm{mmHg}$ or antihypertensive drug treatment in a patient with a history of hypertension); (5) raised FPG ( $\geq 100 \mathrm{mg} / \mathrm{dL}$, or drug treatment of elevated glucose).

\subsection{Anthropomorphic and Blood Chemistry Measurement}

Trained health workers or nurses measured height, weight, WC, and BP following standardized procedures. Height was measured to the nearest $0.1 \mathrm{~cm}$ using a height tape (model 206, SECA). With the participant standing and wearing a single layer of clothing, weight was measured to the nearest $0.1 \mathrm{~kg}$ using a body fat meter (BC601, TANITA). WC was measured in centimeters at the midway between the lowest rib margin and the top of the iliac crest using a SECA tape measure. BP was measured at least three times using a standard mercury sphygmomanometer after the participants rested at least five minutes in a seated position. SBP was measured at the first appearance of a pulse sound (Korotkoff phase 1) and DBP at the disappearance of the pulse sound (Korotkoff phase 5). We used the mean of three satisfactory measurements for analyses.

Trained nurses collected overnight fasting blood samples. Plasma and serum samples were frozen and stored at -86 degrees centigrade for later laboratory analysis. All samples were analyzed in a national lab in Beijing with strict quality control. FPG was measured by the GOD-PAP (Randox Laboratories Ltd., London, UK). The concentration of serum HDL-C and TG were measured by the enzymatic method and CHOD-PAP (Kyowa Medex Co., Ltd, Tokyo, Japan), respectively [27].

\subsection{Assessment of Covariates}

Trained interviewers used standard questionnaires to collect sociodemographic characteristics, smoking, drinking, physical activity, medical history, annual family income, and community information. We grouped participants into three age categories (18-44, 45-59, and 60-75 years). Marital status was categorized into two statuses (single and married). We calculated the family incomes according to household size and grouped them into low, middle and high. We calculated the community urbanicity index based on 12 multidimensional components reflecting the heterogeneity in economic, social, demographic, and infrastructural characteristics at the community level [28], and grouped the urbanicity into low, middle and high. Physical activity includes four domains: occupational, household chore, leisure time, and transportation activities. Participants reported all activities in average hours per week, and we converted the time spent in each activity into a metabolic equivalent of task (MET) hours per week based on the Compendium of Physical Activities. We categorized the total MET hours per week into low, middle and high. We calculated the body mass index (BMI) as measured weight in kilograms divided by measured height in meters squared, and grouped the BMI into thin, normal and overweight. We classified the smoking status as current or ever/never. We categorized the alcohol drinking status as non/ever-drinking and drinking. We also considered the baseline BMI, baseline WC, $\mathrm{BP}, \mathrm{TG}, \mathrm{FPG}$, and HDL-C as potential confounders.

\subsection{Statistical Analysis}

Due to regional disparities in dietary intakes, we performed all analyses separately for the northern and southern regions based on statistically significant $(p<0.05)$ interactions between regions and each cereal subtype, and because of geographic variations in cooking and diet in China. For the baseline characteristics of the participants, we expressed categorical variables as numbers (percentages) and 
examined them with a chi-square test. We expressed continuous variables with normal distribution as means \pm standard deviation and used the z-test. We presented skewed distribution variables presented as medians (interquartile ranges) and used non-parametric statistical hypothesis test including the Wilcoxon signed-rank test and the Kruskal-Wallis test. The Cochran and Mantel-Haenszel and Hierarchical Analysis of Variance were used to analyze the baseline characteristics of normal and developed into MetS in 2015 by regions. We grouped the baseline rice, wheat and wheat products, coarse cereal, and tuber consumption into quartiles by region, and constructed a series of multivariable logistic regression models to assess regional disparities in the association of different levels of consumption of each cereal with the prevalence of MetS. We also tested linear trends by assigning median values to quartiles of consumption of each cereal and modeled this variable as a continuous term. After a Box-Cox transformation, the continuous variables still showed a skewed distribution. Therefore, we estimated the association between the consumption of each cereal subtype and the risk of MetS sensitivity factors WC, BP, TG, FPG, and HDL-C with quantile regression models adjusted for baseline covariates. We conducted all statistical analyses using the SAS 9.4 (SAS Institute, Inc., Cary, NC, USA).

\section{Results}

\subsection{Baseline Characteristics of the Study Population and Cereal Consumption}

Of the 2892 participants included in the study, 1088 were northern region $(47.24 \%$ from men and $52.76 \%$ from women), 1804 were southern region (49.28\% from men and $50.72 \%$ from women). Table 1 shows the baseline characteristics by regions. We found significant differences between the northern and southern regions in age, urbanicity index, physical activity, and BMI. Northern participants were more likely to have higher BMI, WC, and BP $(p<0.001)$. The TEI median was higher among southern participants than among northern $(p<0.001)$. Table 2 shows that the prevalence of MetS in northern China $32.5 \%$ was higher than that in southern China $20.7 \%$.

Table 1. Baseline characteristics of the study population by regions.

\begin{tabular}{|c|c|c|c|}
\hline Factors & Northern & Southern & $p$-Value \\
\hline Gender, $n(\%)$ & & & 0.2884 \\
\hline Men & $514(47.24)$ & $889(49.28)$ & \\
\hline Women & $574(52.76)$ & $915(50.72)$ & \\
\hline \multicolumn{4}{|l|}{ Age (\%), $n$} \\
\hline 18-44 years & $413(37.96)$ & $631(34.98)$ & 0.0325 \\
\hline $45-59$ years & $334(30.7)$ & $504(27.94)$ & \\
\hline 60-years & $341(31.34)$ & $669(37.08)$ & \\
\hline Marital status, $n(\%)$ & & & 0.1024 \\
\hline Single & $86(7.9)$ & $175(9.7)$ & \\
\hline Married & $1002(92.1)$ & $1629(90.3)$ & \\
\hline \multicolumn{4}{|l|}{ Urbanicity index, $n(\%)$} \\
\hline Low & $454(41.73)$ & $506(28.05)$ & $<.0001$ \\
\hline Middle & $346(31.8)$ & $621(34.42)$ & \\
\hline High & $288(26.47)$ & $677(37.53)$ & \\
\hline Income, $n(\%)$ & & & 0.1180 \\
\hline Low & $353(32.5)$ & 604(33.84) & \\
\hline Middle & $346(31.86)$ & $611(34.23)$ & \\
\hline High & $387(35.64)$ & $570(31.93)$ & \\
\hline Physical activity, $n(\%)$ & & & 0.0009 \\
\hline Low & $331(30.42)$ & 633(35.09) & \\
\hline Middle & $350(32.17)$ & $615(34.09)$ & \\
\hline High & $407(37.41)$ & $556(30.82)$ & \\
\hline Smoking, $n(\%)$ & & & 0.9334 \\
\hline Ever/Never & $723(66.45)$ & $1202(66.63)$ & \\
\hline Current & $364(33.46)$ & $601(33.31)$ & \\
\hline Alcohol, $n(\%)$ & & & 0.6489 \\
\hline Ever/Never & $709(65.17)$ & $1158(64.19)$ & \\
\hline Current & $379(34.83)$ & $645(35.75)$ & \\
\hline
\end{tabular}


Table 1. Cont.

\begin{tabular}{lccc}
\hline Factors & Northern & Southern & $p$-Value \\
\hline BMI, $n(\%)$ & & & $<0.0001$ \\
Thin & $59(5.42)$ & $165(9.15)$ & \\
$\quad$ Normal & $750(68.93)$ & $1308(72.51)$ & \\
Overweight & $279(25.64)$ & $331(18.35)$ & \\
BMI $\left(\mathrm{kg} / \mathrm{m}^{2}\right)$ & $22.99(21.12,25.07)$ & $22.04(20.28,24.16)$ & $<0.0001$ \\
WC $(\mathrm{cm})$ & $81(76,87)$ & $79(73,85)$ & 0.0003 \\
SBP $(\mathrm{mmHg})$ & $120(111,126)$ & $118(109,126)$ & 0.0003 \\
DBP $(\mathrm{mmHg})$ & $80(75,82)$ & $77(70,81)$ & $<0.0001$ \\
HDL-C $(\mathrm{mg} / \mathrm{dL})$ & $54(45,64.5)$ & $55(46,65)$ & 0.0771 \\
TG $(\mathrm{mg} / \mathrm{dL})$ & $105(73,162)$ & $101(71,151)$ & 0.1422 \\
FPG $(\mathrm{mg} / \mathrm{dL})$ & $90(83,99)$ & $92(85,100)$ & 0.0457 \\
TEI $(\mathrm{kcal} / \mathrm{day})$ & $2116.53(1757.5,2519.75)$ & $2247.34(1837.91,2740.79)$ & $<0.0001$ \\
\hline
\end{tabular}

Data of categorical variables expressed as number (\%); Medians (interquartile ranges) for skewed parameters.

Table 2. Baseline characteristics of normal and developed into MetS in 2015 by regions.

\begin{tabular}{|c|c|c|c|c|c|}
\hline \multirow{2}{*}{ Factors } & \multicolumn{2}{|c|}{ Northern } & \multicolumn{2}{|c|}{ Southern } & \multirow{2}{*}{$p$-Value } \\
\hline & Normal & MetS & Normal & MetS & \\
\hline Case, $n(\%)$ & $734(67.46)$ & $354(32.54)$ & $1430(79.27)$ & $374(20.73)$ & $<0.0001$ \\
\hline Gender, $n(\%)$ & & & & & $<0.0001$ \\
\hline Men & 739(51.68) & 150(40.11) & $363(49.46)$ & $151(42.66)$ & \\
\hline Women & $691(48.32)$ & $224(59.89)$ & $371(50.54)$ & 203(57.34) & \\
\hline Age, $n(\%)$ & & & & & $<0.0001$ \\
\hline 18-44 years & $543(37.97)$ & $88(23.53)$ & $326(44.41)$ & $87(24.58)$ & \\
\hline $45-59$ years & $385(26.92)$ & $119(31.82)$ & $203(27.66)$ & $131(37.01)$ & \\
\hline 60- years & $502(35.1)$ & $167(44.65)$ & $205(27.93)$ & $136(38.42)$ & \\
\hline Marital status, $n(\%)$ & & & & & 0.848 \\
\hline Single & $136(9.51)$ & $39(10.43)$ & $62(8.45)$ & $24(6.78)$ & \\
\hline Married & 1294(90.49) & $335(89.57)$ & $672(91.55)$ & $330(93.22)$ & \\
\hline Urbanicity index, $n(\%)$ & & & & & $<0.0001$ \\
\hline Low & $421(29.44)$ & $85(22.73)$ & $321(43.73)$ & $133(37.57)$ & \\
\hline Middle & $494(34.55)$ & $127(33.96)$ & $238(32.43)$ & $108(30.51)$ & \\
\hline High & $515(36.01)$ & $162(43.32)$ & $175(23.84)$ & $113(31.92)$ & \\
\hline Income, $n(\%)$ & & & & & 0.8334 \\
\hline Low & $482(34.06)$ & $122(32.97)$ & $242(33.06)$ & $111(31.36)$ & \\
\hline Middle & $486(34.35)$ & $125(33.78)$ & 221(30.19) & $125(35.31)$ & \\
\hline High & $447(31.59)$ & $123(33.24)$ & $269(36.75)$ & $118(33.33)$ & \\
\hline Physical activity, $n(\%)$ & & & & & 0.0007 \\
\hline Low & $484(33.85)$ & $149(39.84)$ & $200(27.25)$ & 131(37.01) & \\
\hline Middle & $505(35.31)$ & $110(29.41)$ & $237(32.29)$ & $113(31.92)$ & \\
\hline High & $441(30.84)$ & $115(30.75)$ & 297(40.46) & $110(31.07)$ & \\
\hline Smoking, $n(\%)$ & & & & & 0.0001 \\
\hline Ever/Never & $927(64.83)$ & $275(73.53)$ & $471(64.17)$ & 252(71.19) & \\
\hline Current & $502(35.1)$ & $99(26.47)$ & $262(35.69)$ & $102(28.81)$ & \\
\hline Alcohol, $n(\%)$ & & & & & 0.0162 \\
\hline Ever/Never & $894(62.52)$ & 264(70.59) & $476(64.85)$ & $233(65.82)$ & \\
\hline Current & $535(37.41)$ & $110(29.41)$ & $258(35.15)$ & $121(34.18)$ & \\
\hline BMI, $n(\%)$ & & & & & $<0.0001$ \\
\hline Thin & $155(10.84)$ & $10(2.67)$ & $54(7.36)$ & $5(1.41)$ & \\
\hline Normal & $1101(76.99)$ & $207(55.35)$ & $554(75.48)$ & $196(55.37)$ & \\
\hline Overweight & 174(12.17) & 157(41.98) & 126(17.17) & $153(43.22)$ & \\
\hline BMI $\left(\mathrm{kg} / \mathrm{m}^{2}\right)$ & $21.57(19.91,23.49)$ & $24.31(22.27,26.21)$ & $22.2(20.52,24.24)$ & $24.61(22.66,26.55)$ & $<0.0001$ \\
\hline$W C(\mathrm{~cm})$ & $77(71,83)$ & $85(79,90)$ & $80(74,85)$ & $85(79,90)$ & $<0.0001$ \\
\hline $\mathrm{SBP}(\mathrm{mmHg})$ & $117(108,125)$ & $120(113,129)$ & $119(110,125)$ & $121(117,129)$ & $<0.0001$ \\
\hline DBP (mmHg) & $76(70,81)$ & $79(73,83)$ & $80(72,82)$ & $80(79,83)$ & $<0.0001$ \\
\hline HDL-C (mmol/L) & $56(47,66)$ & $53(44,61)$ & $55(47,65)$ & $51(43,63)$ & $<0.0001$ \\
\hline $\mathrm{TG}(\mathrm{mmol} / \mathrm{L})$ & $96(67,143)$ & $119(82,181)$ & $95(66,146)$ & $125(89,189)$ & $<0.0001$ \\
\hline Glucose (mmol/L) & $91(84,98)$ & $94.5(88,103)$ & $89(82,98)$ & $94(86,102)$ & $<0.0001$ \\
\hline
\end{tabular}

Table 3 shows the baseline characteristics of food consumption according to the medians and interquartile ranges. The monthly consumption of rice in the southern region $(9.00 \mathrm{~kg} / \mathrm{month})$ was more than twice that in the northern region $(3.60 \mathrm{~kg} / \mathrm{month})$, and the monthly consumption of wheat and wheat products in northern region $(4.20 \mathrm{~kg} / \mathrm{month})$ was more than twice that in the southern region $(1.50 \mathrm{~kg} / \mathrm{month})$. The medians of rice, vegetable and red meat consumption were higher in the 
southern region $(p<0.05)$, while the medians of wheat and wheat products, coarse cereal, tuber and fruit consumption were higher in the northern region $(p<0.001)$.

Table 3. Baseline characteristics of monthly food consumption by regions ( $\mathrm{kg} / \mathrm{month})$.

\begin{tabular}{lccccccc}
\hline \multirow{2}{*}{ Subgroups } & \multicolumn{3}{c}{ Northern } & \multicolumn{3}{c}{ Southern } & $p$-Value \\
\cline { 2 - 7 } & Median & $\mathbf{P}_{\text {25th }}$ & $\mathbf{P}_{\text {75th }}$ & Median & $\mathbf{P}_{\text {25th }}$ & $\mathbf{P}_{\text {75th }}$ & \\
\hline Rice & 3.60 & 0.72 & 6.00 & 9.00 & 6.00 & 11.70 & $<0.0001$ \\
Wheat and products & 4.20 & 1.77 & 7.28 & 1.50 & 0.56 & 3.05 & $<0.0001$ \\
Coarse cereals & 0.50 & 0.23 & 1.04 & 0.20 & 0.08 & 0.50 & $<0.0001$ \\
Tuber & 0.80 & 0.33 & 1.60 & 0.30 & 0.10 & 0.60 & $<0.0001$ \\
Vegetable & 5.73 & 3.34 & 8.80 & 6.00 & 3.58 & 9.35 & 0.043 \\
Fruit & 2.40 & 1.25 & 4.86 & 1.78 & 0.81 & 3.45 & $<0.0001$ \\
Red meat & 0.98 & 0.49 & 1.83 & 1.64 & 0.85 & 3.00 & $<0.0001$ \\
\hline
\end{tabular}

Abbreviation: $\mathrm{P}=$ percentile.

\subsection{The Association between Cereal Subtype Intake and Risk of MetS}

Table 4 shows the southern region risk of MetS per quartiles of monthly consumption of each cereal, and Table 5 shows the northern region risk. The risk of MetS decreased as the monthly consumption of rice increased in the southern region. After adjustment for confounding factors (gender; age; marital status; urbanicity index; income level; BMI, smoking; alcohol; physical activity, TEI; vegetable; fruit; red meats), the second and the fourth quartiles of rice consumption were inversely associated with the prevalence of MetS $(p<0.05)$, wheat and wheat products intake were positively associated with the risk of MetS $(p<0.05)$, coarse cereals and tuber intake had no association with the risk of MetS in the southern region. In the northern region, rice, wheat and wheat products, coarse cereals and tuber intake showed no association with the risk of MetS.

Table 4. The association between cereal subtype intakes and risk of metabolic syndrome among adults in the southern area.

\begin{tabular}{|c|c|c|c|c|c|}
\hline Subgroups & Q1 & Q2 & Q3 & Q4 & $p$-Value \\
\hline \multicolumn{6}{|l|}{ Rice } \\
\hline Participants & 252 & 603 & 495 & 454 & \\
\hline Median (kg/month) & 3.66 & 6.26 & 9.19 & 14.4 & \\
\hline Model1 & Ref & $0.716(0.505,1.015)$ & $0.796(0.552,1.147)$ & $0.689(0.470,1.010)$ * & 0.0164 \\
\hline Model2 & Ref & $0.611(0.422,0.885)$ * & $0.732(0.499,1.075)$ & $0.646(0.430,0.971)$ * & 0.2841 \\
\hline Model3 & Ref & $0.635(0.432,0.934)$ * & $0.744(0.496,1.115)$ & $0.709(0.458,1.003)$ * & 0.4641 \\
\hline \multicolumn{6}{|l|}{ Wheat and products } \\
\hline Participants & 450 & 453 & 452 & 449 & \\
\hline Median (kg/month) & 0.23 & 0.99 & 2.26 & 6.42 & \\
\hline Model1 & Ref & $1.860(1.310,2.641) *$ & $1.678(1.167,2.412)^{*}$ & $1.979(1.379,2.841)$ * & 0.0449 \\
\hline Model2 & Ref & $1.641(1.131,2.381) *$ & $1.496(1.020,2.195) *$ & $1.800(1.231,2.632) *$ & 0.0130 \\
\hline Model3 & Ref & $1.601(1.092,2.346)$ * & $1.479(0.997,2.192)$ * & $1.925(1.292,2.867)^{*}$ & 0.0146 \\
\hline \multicolumn{6}{|l|}{ Coarse cereals } \\
\hline Participants & 449 & 476 & 435 & 444 & \\
\hline Median (kg/month) & 0.02 & 0.14 & 0.34 & 1.72 & \\
\hline Model1 & Ref & $0.934(0.664,1.314)$ & $1.199(0.853,1.685)$ & $1.184(0.837,1.676)$ & 0.2862 \\
\hline Model2 & Ref & $1.011(0.705,1.451)$ & $1.225(0.852,1.761)$ & $1.257(0.869,1.819)$ & 0.3987 \\
\hline Model3 & Ref & $1.015(0.700,1.471)$ & $1.185(0.815,1.722)$ & $1.283(0.866,1.899)$ & 0.2467 \\
\hline \multicolumn{6}{|l|}{ Tuber } \\
\hline Participants & 379 & 513 & 434 & 478 & \\
\hline Median (kg/month) & 0.02 & 0.16 & 0.38 & 1.29 & \\
\hline Model1 & Ref & $0.989(0.704,1.388)$ & $0.767(0.534,1.100)$ & $0.837(0.589,1.191)$ & 0.6631 \\
\hline Model2 & Ref & $0.924(0.646,1.322)$ & $0.745(0.510,1.088)$ & $0.834(0.576,1.207)$ & 0.4796 \\
\hline Model3 & Ref & $0.979(0.677,1.415)$ & $0.809(0.549,1.192)$ & $0.826(0.561,1.216)$ & 0.4168 \\
\hline
\end{tabular}

Abbreviation: $\mathrm{Q}=$ quarter. Data intake expressed as median (25th percentile, 75th percentile); Model1: Crude; Model2: adjusted gender, age, marital status, income level, urbanicity index; Model3: model 2+body mass index, smoking, alcohol, physical activity, TEI, vegetable, fruit, red meat consumption, and other type of cereals intake. ${ }^{*} p<0.05$. 
Table 5. The association between cereal subtype intakes and risk of metabolic syndrome among adults in northern area.

\begin{tabular}{|c|c|c|c|c|c|}
\hline Subgroups & Q1 & Q2 & Q3 & Q4 & $p$-Value \\
\hline \multicolumn{6}{|l|}{ Rice } \\
\hline Participants & 271 & 274 & 283 & 260 & \\
\hline Median (kg/month) & 0.36 & 2.01 & 5.44 & 11.77 & \\
\hline Model1 & Ref & $0.789(0.539,1.153)$ & $0.933(0.625,1.393)$ & $0.998(0.649,1.535)$ & 0.5702 \\
\hline Model2 & Ref & $0.779(0.520,1.166)$ & $0.966(0.631,1.480)$ & $1.059(0.669,1.676)$ & 0.3811 \\
\hline Model3 & Ref & $0.690(0.457,1.042)$ & $0.900(0.583,1.389)$ & $0.981(0.610,1.578)$ & 0.5617 \\
\hline \multicolumn{6}{|l|}{ Wheat and products } \\
\hline Participants & 272 & 274 & 270 & 272 & \\
\hline Median (kg/month) & 1.04 & 2.97 & 5.71 & 13.75 & \\
\hline Model1 & Ref & $0.974(0.663,1.431)$ & $1.006(0.681,1.487)$ & $1.073(0.705,1.635)$ & 0.5198 \\
\hline Model2 & Ref & $0.929(0.617,1.400)$ & $0.949(0.625,1.441)$ & $1.008(0.642,1.582)$ & 0.7345 \\
\hline Model3 & Ref & $0.930(0.614,1.410)$ & $0.964(0.631,1.473)$ & $0.918(0.576,1.464)$ & 0.7887 \\
\hline \multicolumn{6}{|l|}{ Coarse cereals } \\
\hline Participants & 272 & 278 & 267 & 271 & \\
\hline Median (kg/month) & 0.10 & 0.37 & 0.74 & 3.84 & \\
\hline Model1 & Ref & $1.049(0.728,1.510)$ & $0.755(0.518,1.100)$ & $0.797(0.543,1.170)$ & 0.1416 \\
\hline Model2 & Ref & $1.046(0.707,1.548)$ & $0.764(0.510,1.145)$ & $0.780(0.518,1.175)$ & 0.1350 \\
\hline Model3 & Ref & $1.059(0.712,1.576)$ & $0.756(0.503,1.137)$ & $0.721(0.469,1.109)$ & 0.0422 \\
\hline \multicolumn{6}{|l|}{ Tuber } \\
\hline Participants & 272 & 237 & 344 & 235 & \\
\hline Median (kg/month) & 0.14 & 0.48 & 1.16 & 3.68 & \\
\hline Model1 & Ref & $0.877(0.601,1.278)$ & $0.704(0.494,1.004)$ & $0.724(0.483,1.084)$ & 0.1556 \\
\hline Model2 & Ref & $0.910(0.608,1.364)$ & $0.801(0.549,1.167)$ & $0.787(0.507,1.222)$ & 0.3035 \\
\hline Model3 & Ref & $0.843(0.559,1.273)$ & $0.758(0.516,1.115)$ & $0.724(0.461,1.139)$ & 0.2137 \\
\hline
\end{tabular}

Abbreviation: $\mathrm{Q}=$ quarter. Data intake expressed as median (25th percentile, 75th percentile); Model1: Crude; Model2: adjusted gender, age, marital status, income level, urbanicity index; Model3: model 2+body mass index, smoking, alcohol, physical activity, TEI, vegetable, fruit, red meat consumption and other type of cereals intake.

\subsection{The Association between Cereal Subtypes Intake and the Risk of Individual Components of MetS}

Table 6 shows the individual components of the MetS quantile regression results among northern adults. The monthly consumption of rice showed a significant positive association with WC across the entire conditional WC distribution $(p<0.05)$, with the lowest WC association at the 25th quantile, the highest at the 90th quantile $(0.197,0.240,0.258$ and 0.357 at the 25th, 50th, 75th and 90th quantiles, respectively). Rice consumption in the northern region showed a negative association with FPG $(-0.036,-0.037$ and -0.023 at the 10th, 25th, and 50th quantiles, respectively). The monthly intake of wheat and wheat products was positively associated with FPG and HDL-C, at the lower end of the conditional distribution of FPG (0.010 and 0.012 at the 10th and 50th quantiles, respectively) and HDL-C (0.005 and 0.003 at the 10th, and 25th quantiles, respectively).

Table 7 shows the individual components of the MetS quantile regression results among southern adults. Monthly consumption of rice was negatively associated with WC, SBP, and HDL-C, and the coefficients were statistically significant at the conditional distribution of WC (-0.105 at the 25th quantile), SBP $(-0.259$ at the 75 th quantile). The wheat and wheat products consumption showed an inverse relationship with FPG across the entire conditional distribution $(-0.020,-0.024,-0.028,-0.026$ and -0.030 at the 10th, 25th, 50th, 75 th, and 90th quantiles, respectively). Coarse cereals consumption had a positive relationship with WC, SBP, FPG and HDL-C, and the coefficients were statistically significant at the conditional distribution of WC $(0.836$ at the 90th quantile), SBP $(0.915,0.603$ at the 10th and 25th quantile, respectively), HDL-C $(0.445,0.53,0.551$ at the 10 th, 25 th and 50 th, respectively). The tuber intake showed an inversely associated with SBP and FPG, and the coefficients were statistically significant at the conditional distribution of FPG $(-0.157,-0.105$ and -0.076 at the 10th, 25th and 50th, respectively). 
Table 6. Coefficient estimates from a quantile regression on individual components of MetS among northern China by cereal subtype intake.

\begin{tabular}{|c|c|c|c|c|c|c|}
\hline \multirow{2}{*}{ Variable } & & \multicolumn{5}{|c|}{ Quantile $^{\#}$} \\
\hline & & 10th & 25th & 50th & 75th & 90th \\
\hline \multicolumn{7}{|l|}{ WC } \\
\hline & Rice & $0.110(-0.079,0.298)$ & $0.197(0.047,0.347) *$ & $0.240(0.071,0.409) *$ & $0.258(0.091,0.425) *$ & $0.357(0.127,0.586)$ * \\
\hline & Wheat and products & $-0.004(-0.210,0.202)$ & $-0.016(-0.124,0.091)$ & $-0.027(-0.138,0.085)$ & $0.030(-0.105,0.165)$ & $0.160(-0.038,0.358)$ \\
\hline & Coarse cereals & $0.005(-0.242,0.251)$ & $-0.033(-0.191,0.124)$ & $-0.056(-0.256,0.144)$ & $-0.066(-0.313,0.181)$ & $-0.102(-0.418,0.215)$ \\
\hline & Tuber & $0.236(-0.232,0.704)$ & $-0.026(-0.362,0.309)$ & $0.177(-0.186,0.539)$ & $0.066(-0.341,0.473)$ & $-0.057(-0.733,0.619)$ \\
\hline \multicolumn{7}{|l|}{ SBP } \\
\hline & Wheat and products & $-0.084(-0.306,0.137)$ & $-0.010(-0.214,0.193)$ & $-0.104(-0.248,0.040)$ & $\begin{array}{l}0.115(-0.210,0.440) \\
-0.072(-0.363,0.219)\end{array}$ & $\begin{array}{l}0.144(-0.327,0.013) \\
0.206(-0.282,0.695)\end{array}$ \\
\hline & Coarse cereals & $0.090(-0.405,0.585)$ & $0.069(-0.295,0.434)$ & $0.046(-0.230,0.322)$ & $-0.004(-0.316,0.308)$ & $-0.050(-0.489,0.388)$ \\
\hline & Tuber & $-0.785(-1.920,0.351)$ & $-0.439(-1.349,0.471)$ & $-0.455(-1.216,0.307)$ & $-0.600(-1.336,0.135)$ & $-0.012(-1.686,1.662)$ \\
\hline \multicolumn{7}{|c|}{ 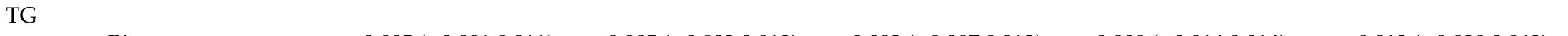 } \\
\hline & Rice & $0.005(-0.001,0.011)$ & $0.005(-0.002,0.013)$ & $0.002(-0.007,0.012)$ & $0.000(-0.014,0.014)$ & $0.012(-0.020,0.043)$ \\
\hline & Wheat and products & $0.002(-0.003,0.007)$ & $-0.001(-0.007,0.004)$ & $-0.005(-0.013,0.004)$ & $-0.006(-0.014,0.001)$ & $-0.013(-0.023,-0.002)$ * \\
\hline & Coarse cereals & $0.000(-0.009,0.008)$ & $0.000(-0.008,0.007)$ & $0.000(-0.010,0.010)$ & $-0.001(-0.016,0.014)$ & $-0.005(-0.058,0.049)$ \\
\hline & Tuber & $0.012(0.001,0.024)^{*}$ & $0.007(-0.009,0.023)$ & $-0.001(-0.021,0.019)$ & $-0.004(-0.037,0.028)$ & $-0.031(-0.082,0.019)$ \\
\hline \multicolumn{7}{|l|}{ FPG } \\
\hline & Rice & $\begin{array}{c}-0.036 \\
(-0.053,-0.018) \text { * }\end{array}$ & $\begin{array}{c}-0.037 \\
(-0.053,-0.021) *\end{array}$ & $\begin{array}{c}-0.023 \\
(-0.035,-0.012) \text { * }\end{array}$ & $-0.007(-0.023,0.009)$ & $0.015(-0.010,0.039)$ \\
\hline & Wheat and products & $0.010(0.004,0.016)$ * & $0.007(-0.002,0.015)$ & $0.012(0.004,0.020)$ * & $0.012(-0.001,0.025)$ & $0.024(-0.008,0.055)$ \\
\hline & Coarse cereals & $0.009(-0.018,0.035)$ & $0.008(-0.011,0.027)$ & $0.004(-0.011,0.019)$ & $0.000(-0.024,0.024)$ & $-0.007(-0.054,0.042)$ \\
\hline & Tuber & $0.007(-0.042,0.057)$ & $-0.006(-0.042,0.03)$ & $-0.004(-0.024,0.016)$ & $-0.004(-0.047,0.039)$ & $0.032(-0.053,0.117)$ \\
\hline \multicolumn{7}{|l|}{ HDL-C } \\
\hline & Rice & $-0.002(-0.010,0.006)$ & $-0.001(-0.006,0.004)$ & $-0.002(-0.006,0.002)$ & $-0.002(-0.007,0.003)$ & $0.000(-0.007,0.007)$ \\
\hline & Wheat and products & $0.005(0.001,0.009) *$ & $0.003(0.000,0.007) *$ & $0.002(-0.001,0.004)$ & $0.003(-0.002,0.008)$ & $0.004(-0.004,0.011)$ \\
\hline & Coarse cereals & $0.003(-0.018,0.023)$ & $0.002(-0.004,0.007)$ & $0.001(-0.004,0.006)$ & $0.001(-0.006,0.007)$ & $-0.001(-0.016,0.015)$ \\
\hline & Tuber & $-0.005(-0.024,0.015)$ & $-0.002(-0.016,0.012)$ & $-0.001(-0.014,0.011)$ & $0.005(-0.008,0.019)$ & $0.014(-0.003,0.031)$ \\
\hline
\end{tabular}

fruit, red meat consumption, and other type of cereals intake. ${ }^{\#}$ Coefficient $(95 \% \mathrm{CI}) ;{ }^{*} p<0.05$. 
Table 7. Coefficient estimates from a quantile regression on individual components of MetS among southern China by cereals consumption.

\begin{tabular}{|c|c|c|c|c|c|c|}
\hline \multirow{2}{*}{ Variable } & & \multicolumn{5}{|c|}{ Quantile \# } \\
\hline & & 10th & 25th & 50th & 75th & 90th \\
\hline \multicolumn{7}{|l|}{ WC } \\
\hline & Rice & $-0.011(-0.168,0.146)$ & $-0.105(-0.203,-0.008)$ * & $-0.062(-0.185,0.06)$ & $-0.067(-0.183,0.049)$ & $-0.087(-0.237,0.063)$ \\
\hline & Wheat and products & $-0.059(-0.203,0.085)$ & $-0.018(-0.122,0.086)$ & $0.033(-0.074,0.139)$ & $0.215(0.024,0.406) *$ & $0.161(-0.158,0.481)$ \\
\hline & Coarse cereals & $0.271(-0.119,0.660)$ & $0.256(-0.078,0.589)$ & $0.197(-0.162,0.557)$ & $0.140(-0.518,0.799)$ & $0.836(0.065,1.608)^{*}$ \\
\hline & Tuber & $-0.187(-0.724,0.350)$ & $-0.232(-0.920,0.457)$ & $-0.019(-0.583,0.545)$ & $0.174(-0.611,0.959)$ & $0.363(-0.607,1.333)$ \\
\hline \multicolumn{7}{|c|}{ 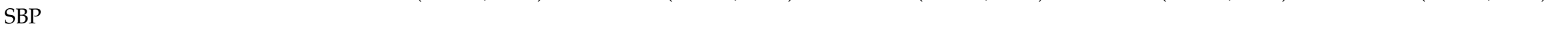 } \\
\hline & Rice & $-0.025(-0.259,0.209)$ & $-0.056(-0.268,0.156)$ & $-0.038(-0.24,0.163)$ & $-0.209(-0.437,0.018)$ & $-0.254(-0.608,0.100)$ \\
\hline & Wheat and products & $-0.083(-0.324,0.158)$ & $-0.176(-0.440,0.089)$ & $-0.275(-0.586,0.036)$ & $0.045(-0.280,0.369)$ & $0.030(-0.424,0.484)$ \\
\hline & Coarse cereals & $0.915(0.422,1.407) *$ & $0.603(0.155,1.051)$ * & $0.054(-0.506,0.614)$ & $-0.155(-1.264,0.955)$ & $-0.084(-1.638,1.471)$ \\
\hline & Tuber & $-0.516(-2.584,-0.448)$ * & $-0.354(-1.935,1.226)$ & $0.335(-0.841,1.511)$ & $-0.136(-1.341,1.069)$ & $-0.648(-2.408,1.113)$ \\
\hline \multicolumn{7}{|c|}{ 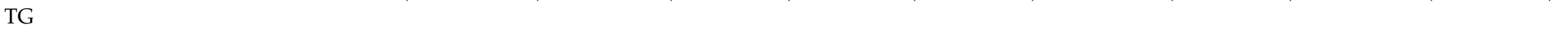 } \\
\hline & Rice & $-0.001(-0.006,0.004)$ & $0.001(-0.005,0.007)$ & $-0.001(-0.009,0.007)$ & $0.003(-0.012,0.018)$ & $-0.001(-0.022,0.019)$ \\
\hline & Wheat and products & $-0.005(-0.012,0.003)$ & $-0.005(-0.011,0.002)$ & $-0.005(-0.015,0.005)$ & $-0.008(-0.024,0.008)$ & $-0.007(-0.032,0.017)$ \\
\hline & Coarse cereals & $0.001(-0.031,0.032)$ & $0.003(-0.026,0.033)$ & $-0.010(-0.040,0.019)$ & $-0.004(-0.046,0.038)$ & $-0.037(-0.124,0.050)$ \\
\hline & Tuber & $0.024(-0.019,0.067)$ & $0.019(-0.029,0.066)$ & $0.036(-0.009,0.081)$ & $0.016(-0.049,0.082)$ & $-0.047(-0.147,0.053)$ \\
\hline \multicolumn{7}{|c|}{ 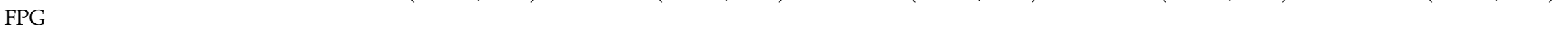 } \\
\hline & Rice & $0.013(0.002,0.023) *$ & $0.004(-0.005,0.013)$ & $-0.006(-0.016,0.005)$ & $-0.002(-0.017,0.013)$ & $-0.008(-0.034,0.019)$ \\
\hline & Wheat and products & $-0.020(-0.039,0.000)$ * & $-0.024(-0.039,-0.01) *$ & $-0.028(-0.043,-0.014)$ * & $-0.026(-0.043,-0.008) *$ & $-0.030(-0.050,-0.011)$ * \\
\hline & Coarse cereals & $0.053(-0.005,0.111)$ & $0.025(-0.015,0.064)$ & $0.000(-0.030,0.031)$ & $-0.005(-0.045,0.036)$ & $-0.005(-0.072,0.061)$ \\
\hline & Tuber & $-0.157(-0.259,-0.054) *$ & $-0.105(-0.174,-0.035)$ * & $-0.076(-0.128,-0.023)$ * & $-0.041(-0.126,0.043)$ & $-0.029(-0.140,0.083)$ \\
\hline \multicolumn{7}{|l|}{ HDL-C } \\
\hline & Rice & $-0.084(-0.256,0.089)$ & $-0.037(-0.165,0.091)$ & $-0.094(-0.219,0.032)$ & $-0.087(-0.237,0.063)$ & $-0.013(-0.183,0.157)$ \\
\hline & Wheat and products & $0.035(-0.147,0.218)$ & $0.148(-0.026,0.322)$ & $0.109(-0.055,0.273)$ & $0.180(-0.043,0.403)$ & $0.169(-0.147,0.486)$ \\
\hline & Coarse cereals & $0.445(0.006,0.883)$ * & $0.530(0.125,0.934)$ * & $0.551(0.108,0.993)$ * & $0.593(-0.036,1.222)$ & $0.856(-0.009,1.720)$ \\
\hline & Tuber & $0.324(-0.344,0.992)$ & $0.142(-0.653,0.937)$ & $-0.077(-0.715,0.561)$ & $0.358(-0.860,1.576)$ & $0.901(-0.147,1.949)$ \\
\hline
\end{tabular}

Adjusted gender, age, marital status, income level, urbanicity index, physical activity, drinking, smoking, baseline value of BMI and each homologous MetS component, TEI, vegetable, fruit, red meat consumption, and other type of cereals intake. " Coefficient $(95 \% \mathrm{CI}) ;{ }^{*} p<0.05$ 


\section{Discussion}

Our study involving more than 2892 Chinese individuals without prior MetS or other chronic diseases found rice, wheat and products, coarse cereals and tuber intake disparities in southern and northern China. We found a significant inverse association between rice consumption and MetS, and while it showed a positive association with wheat and wheat products consumption and the prevalence of MetS in southern adults, null associations between each cereal and the risk of MetS were found in northern adults. The associations between cereal subtypes consumption and the risk of the components of MetS varied across the two regions of China.

The association between rice consumption and the prevalence of MetS in southern and northern China is similar to the study from Iran which showed an increased likelihood of T2DM associated with high white rice intake among residents of Tehran (OR: 2.1,95\% CI: 1.1-3.9) and no association in Gorleston [29]. Rice consumption has different effects in different areas. The Korean Genome and Epidemiology Study revealed that the prevalence of MetS was different in men and women with different rice preferences [10]. In Indian and Japan it did not find an association between rice consumption and the prevalence of MetS [9-11,13]. The main reason for this result is likely to be that the rice consumption differed between southern China and northern China. A study in Japan, found that a moderate intake of rice might reduce the risks of incident MetS (HRs: 0.83, 95\% CI: 0.69-0.99) and high BP (HRs: 0.79, 95\% CI: 0.66-0.94) [15]. The Jiangsu Nutrition Study [12], found no significant association between rice intake and incident MetS in a five-year follow-up; rather, the results were in the opposite direction. In the Jiangsu Province in our CHNS southern region, the inconsistency could be partially due to the potential factors. The wheat and wheat products consumption are mostly handmade noodles, steamed bud and handmade dumplings in China, it is much different from western countries. In addition to the wheat and wheat products consumption differed between southern and northern China, the types of wheat and wheat products, which the two regions consumed have also great discrepancy. It mainly consumed the handmade noodles, teamed buns and handmade dumplings in northern China, while people in the southern China mainly consumed processed noodles or pre-packaged pasta. According to the China Food Composition, the sodium content of processed noodle is far more than the handmade noodle [30]. In Japan, wheat consumption reduced the risk of MetS, but the daily intake of wheat products increased the risk of MetS (HRs: 1.19, 95\% CI: 1.05-1.35), and abdominal obesity (HRs: 1.15, 95\% CI: 1.02-1.29) in urban populations [15]. An intervention study showed that the whole grain wheat bread group decreased its visceral fat area, whereas the refined wheat bread group had no significant changes [16]. Therefore, different processing methods of wheat and wheat products may have a great impact on our results.

A cross-sectional study of Chinese adults found that high rice consumption was associated with high TG and low HDL-C in the northern region [31]. The studies in Korea and India showed that TG and HDL-C levels were associated with the white rice intake [8,32,33]. It was inconsistent with our result, since the rice intake in northern adult was low in our study. A randomized trial showed that brown rice and white rice can help reduce $24 \mathrm{~h}$ glucose and fasting insulin responses among overweight Indians [33]. The present study did not show a significant relationship between coarse cereal consumption and the risk of MetS in either the southern or the northern region. A randomized controlled trial indicated that diabetic groups showed marginal decreases in TG and increases in HDL-C with millet consumption, that is, millet was beneficial for T2DM [34]. More research on the relationship between coarse cereals and lipids, and between glucose and HDL-C are needed. A recent meta-analysis also showed that the consumption of whole grain foods compared to refined foods, improved acutely the postprandial glucose and insulin homeostasis in healthy subjects [35]. Consumption of coarse cereals in China is very low and most consumed do not meet the recommended of dietary guidelines. Few studies have focused on Chinese consumption of coarse cereals, such as millet, corn, and sorghum; so, it is difficult to evaluate the relationship between coarse cereal and the risk of MetS.

In Western countries studies mainly find a positive correlation between tuber consumption, obesity, and T2DM [36-38]. However, our results were inconsistent with it, instead showing an inverse 
association between tuber consumption and FPG in southern China. This could be explained by the small amount consumed by our participants. A Japanese cohort study found no relationship between low tuber consumption (1-2 times/week) and CVDs, and a positive association between high tuber consumption ( $\geq 3$ times/week) and CVDs [39]. Another reason for the disparity between China and Western countries could be the different methods of cooking tuber. In China tubers are primarily steamed and boiled, but in Western countries they are mostly fried. The different ways of cooking may affect health differently. Further studies on how cooking methods modify the association between the tuber intake and the risk of MetS are needed.

It suggested that a high consumption of rice might be linked to healthier diet patterns (high fish, seafood and vegetable) in southern China [31]. The inconsistencies of the result between the regions could be due to multiple factors, including different staple food, the amount of cereal consumed, dietary structures, and cooking methods. In addition, a statistical difference in cereal intolerance among the adults in the southern and northern regions of China has also been reported [40]. Our study is the first to examine the association between cereal consumption and MetS in a regional perspective. It mainly studies whether the other food groups and nutrients intake differed between the two regions in the future. This study has several residual confounding factors of an observational study. First, the dietary intake was estimated based on an FFQ that covered the past 12 months of dietary consumption, which may lead to a recall bias, the distinguishability between whole grains and refined grains in our FFQ may not have been clear, and we could not conduct an isocaloric analysis describing results when the total caloric intake is fixed. We used monthly consumptions for episodically consumed foods like coarse cereals and tubers, to relatively stable. Second, measurement errors in the assessments of each cereal consumed are inevitable, and we cannot know the extent to which such an error may bias our results. Third, the sample size of this study is relatively small, containing some of the northern and southern provinces, so we had difficulty generalizing the study results. More studies with larger sample sizes in diverse geographic locations and with longer follow-up periods are needed to examine regional differences.

\section{Conclusions}

In conclusion, our evaluation of the relationship between cereal consumption and the prevalence of MetS and regional disparities in China showed that it had different associations between rice and wheat consumption and a risk of MetS among northern and southern adults. Given the different consumption of staple foods in northern and southern China, regional disparities should be taken into account when studying the relationship between diet and chronic diseases, and making dietary recommendations.

Author Contributions: All authors contributed significantly to this article. L.H. analyzed the data and wrote the first version of the manuscript; H.W., Z.W., J.Z., B.Z. and G.D. performed the surveys; H.W., Z.W. interpreted the results and revised the manuscript; and G.D. critically reviewed the manuscript for important intellectual content. All authors approved the final manuscript.

Funding: The "China Nutritional Transition Cohort Study" project received funding from Ministry of Finance of the People's Republic of China (NO. 13103110700015005). The present study is also sponsored by Carolina Population Center (P2CHD050924, T32 HD007168), the University of North Carolina at Chapel Hill, the NIH (R01-HD30880, DK056350, R24 HD050924, and R01-HD38700) and the NIH Fogarty International Center (D43 TW009077, D43 TW007709) for financial support for the CHNS data collection and analysis files from 1989 to 2015 and future surveys.

Acknowledgments: We are grateful to all the participants in our study and the staff responsible for conducting the China Health and Nutrition Survey. And the authors also acknowledge the team at the National Institute for Nutrition and Health, Chinese Center for Disease Control and Prevention, the Carolina Population Center, University of North Carolina at Chapel Hill.

Conflicts of Interest: The authors declare that they have no conflict of interest regarding the publication of this paper. 


\section{References}

1. GBD 2017 Causes of Death Collaborators. Global, regional, and national age-sex-specific mortality for 282 causes of death in 195 countries and territories, 1980-2017: A systematic analysis for the Global Burden of Disease Study 2017. Lancet 2018. [CrossRef]

2. GBD 2017 Disease and Injury Incidence and Prevalence Collaborators. Global, regional, and national incidence, prevalence, and years lived with disability for 354 diseases and injuries for 195 countries and territories, 1990-2017: A systematic analysis for the Global Burden of Disease Study 2017. Lancet 2018. [CrossRef]

3. Wei-Wei, C.H.; Run-Lin, G.A.; Li-Sheng, L.I.; Man-Lu, Z.H.; Wen, W.A.; Yong-Jun, W.A.; Zhao-Su, W.U.; Hui-Jun, L.I.; Dong-Feng, G.U.; Yue-Jin, Y.A.; et al. Chinese cardiovascular disease report 2017: A summary. Chin. Circ. J. 2018, 33, 1-8. [CrossRef]

4. Alberti, K.; George, M.M.; Zimmet, P.; Shaw, J. The metabolic syndrome-A new worldwide definition. Lancet 2005, 366, 1059-1062. [CrossRef]

5. Reilly, M.P.; Rader, D.J. The metabolic syndrome: More than the sum of its parts? Circulation 2003, 108, 1546-1551. [CrossRef] [PubMed]

6. He, Y.N.; Zhao, W.H.; Zhao, L.Y.; Yu, D.M.; Zhang, J.; Yang, X.G.; Ding, G.G. Prevalence of metabolic syndrome in Chinese adults in 2010-2012. Chin. J. Epidemiol. 2017, 38, 212-215.

7. Bahadoran, Z.; Mirmiran, P.; Delshad, H.; Azizi, F. White rice consumption is a risk factor for metabolic syndrome in Tehrani adults: A prospective approach in Tehran lipid and glucose study. Arch. Iran. Med. 2014, 17, 435-440.

8. Song, S.; Lee, J.; Song, W.; Paik, H.; Song, Y. Carbohydrate intake and refined-grain consumption are associated with metabolic syndrome in the Korean adult population. J. Acad. Nutr. Diet. 2014, 114, 54-62. [CrossRef] [PubMed]

9. Krittanawong, C.; Tunhasiriwet, A.; Zhang, H.; Prokop, L.J.; Chirapongsathorn, S.; Sun, T.; Wang, Z. Is white rice consumption a risk for metabolic and cardiovascular outcomes-A systematic review and meta-analysis. BMJ 2017, 7. [CrossRef] [PubMed]

10. Ahn, Y.; Park, S.; Kwack, H.; Kim, M.K.; Ko, K.; Kim, S.S. Rice-eating pattern and the risk of metabolic syndrome especially waist circumference in Korean Genome and Epidemiology Study (KoGES). BMC Public Health 2013, 13. [CrossRef]

11. Khosravi-Boroujeni, H.; Sarrafzadegan, N.; Mohammadifard, N.; Sajjadi, F.; Maghroun, M.; Asgari, S.; Rafieian-Kopaei, M.; Azadbakht, L. White Rice Consumption and CVD Risk Factors among Iranian Population. J. Health Popul. Nutr. 2013, 31, 252-261. [CrossRef] [PubMed]

12. Shi, Z.; Taylor, A.W.; Hu, G.; Gill, T.; Bch, G.A.W.M.B. Rice intake, weight change and risk of the metabolic syndrome development among Chinese adults: The Jiangsu Nutrition Study (JIN). Asia Pac. J. Clin. Nutr. 2012, 21, 35-43. [CrossRef]

13. Eshak, E.S.; Iso, H.; Yamagishi, K.; Kokubo, Y.; Saito, I.; Yatsuya, H.; Sawada, N.; Inoue, M.; Tsugane, S. Rice consumption is not associated with risk of cardiovascular disease morbidity or mortality in Japanese men and women: A large population-based, prospective cohort study. Am. J. Clin. Nutr. 2014, 100, 199-207. [CrossRef]

14. Hu, E.; Pan, A.; Malik, V.; Sun, Q. White rice consumption and risk of type 2 diabetes: Meta-analysis and systematic review. BMJ 2012, 344, e1454. [CrossRef]

15. Kokubo, Y.; Watanabe, M.; Kobayashi, T.; Miyamoto, Y. Are Japanese Traditional Staple Foods, Rice and Udon Noodle, Increased Risks of Incident Metabolic Syndrome. JACC 2014, 63, 1145-1155. [CrossRef]

16. Kikuchi, Y.; Nozaki, S.; Makita, M.; Yokozuka, S.; Fukudome, S.; Yanagisawa, T.; Aoe, S. Effects of Whole Grain Wheat Bread on Visceral Fat Obesity in Japanese Subjects_ A Randomized Double-Blind Study. Plant. Foods Hum. Nutr. 2018, 73, 161-165. [CrossRef]

17. Šabovič, M.; Lavre, S.; Keber, I. Supplementation of wheat fibre can improve risk profile in patients with dysmetabolic cardiovascular syndrome. Eur. J. Cardiovasc. Prev. Rehabil. 2004, 11, 144-148. [CrossRef]

18. Jones, J.M. Grain-Based Foods and Health. Cereal Foods World 2006. [CrossRef]

19. Kaur, K.D.; Jha, A.; Sabikhi, L.; Singh, A.K. Significance of coarse cereals in health and nutrition: A review. J. Food Sci. Technol. 2014, 51, 1429-1441. [CrossRef]

20. Zheng-Zhu, T.A.; Xing-Le, C.H.; Zhao-Yong, H.U. Research on metabolic syndrome and its diet and economic and behavior factors in guangxi minority municipality. Mod. Pre Med. 2008, 35, 3294-3297. 
21. Hu, Y.I.; Zhai, F. Relatonship Between Metabolic Syndrome and Dietary Modalities in Chinese Population. Pract. Pre Med. 2008, 15, 1-8.

22. Xu, X.; Zhao, L.; Fang, H.; Guo, Q. Cereal intake status and changes of Chinese residents. Food Nutr. C 2017, 23, 44-46.

23. Popkin, B.M.; Du, S.; Zhai, F.; Zhang, B. Cohort Profile: The China Health and Nutrition Surveye monitoring and understanding socio-economic and health change in China, 1989-2011. Int. J. Epidemiol. 2010, 39, 1435-1440. [CrossRef]

24. Zhang, J.; Wang, H.; Wang, Z.; Du, W.; Su, C.; Zhang, J.; Jiang, H.; Jia, X.; Huang, F.; Ouyang, Y.; et al. Prevalence and stabilizing trends in overweight and obesity among children and adolescents in China, 2011-2015. BMC Public Health 2018, 18, 571. [CrossRef] [PubMed]

25. Li, Y.; Song, J.; Pan, H.; Yan, M.; Hu, X.; Ma, G. Valldity of food freouency ouestlonnaire to 1 nvestigate the dletary energy and nutrients intake. Acta Nutr. Sin. 2006, 28, 143-147. [CrossRef]

26. Alberti, K.G.M.M.; Eckel, R.H.; Grundy, S.M.; Zimmet, P.Z.; Cleeman, J.I. Harmonizing the Metabolic Syndrome. Circulation 2009, 120, 1640-1645. [CrossRef] [PubMed]

27. Wang, Z.; Siega-Riz, A.M.; Gordon-Larsen, P.; Cai, J.; Adair, L.S.; Zhang, B.; Popkin, B.M. Diet quality and its association with type 2 diabetes and major cardiometabolic risk factors among adults in China. Nutr. Metab. Cardiovasc. Dis. Nmcd 2018, 28, 987-1001. [CrossRef]

28. Jones-Smith, J.C.; Popkin, B.M. Understanding community context and adult health changes in China: Development of an urbanicity scale. Soc. Sci. Med. 2010, 71, 1436-1446. [CrossRef]

29. Golozar, A.; Khalili, D.; Etemadi, A.; Poustchi, H.; Fazeltabar, A.; Hosseini, F.; Kamangar, F.; Khoshnia, M.; Islami, F.; Hadaegh, F.; et al. White rice intake and incidence of type-2 diabetes: Analysis of two prospective cohort studies from Iran. BMC Public Health 2017, 17, 133. [CrossRef]

30. Yuexin Yang, G.W.; Xingchang, P. China Food Composition; Peking University Medical Press: BeiJing, China, 2009; p. 384.

31. Dong, F.; Howard, A.G.; Herring, A.H.; Popkin, B.M.; Gordon-Larsen, P. White Rice Intake Varies in Its Association with Metabolic Markers of Diabetes and Dyslipidemia Across Region among Chinese Adults. Ann. Nutr. Metab. 2015, 66, 209-218. [CrossRef]

32. Mattei, J.; Hu, F.B.; Campos, H. A higher ratio of beans to white rice is associated with lower cardiometabolic risk factors in Costa Rican adults. Am. J. Clin. Nutr. 2011, 94, 869-876. [CrossRef]

33. Mohan, V.; Spiegelman, D.; Sudha, V.; Gayathri, R.; Hong, B.; Praseena, K.; Anjana, R.M.; Wedick, N.M.; Arumugam, K.; Malik, V.; et al. Effect of brown rice, white rice, and brown rice with legumes on blood glucose and insulin responses in overweight Asian Indians: A randomized controlled trial. Diabetes Technol. Ther. 2014, 16, 317-325. [CrossRef] [PubMed]

34. Ugare, R.; Chimmad, B.; Naik, R.; Bharati, P.; Itagi, S. Glycemic index and significance of barnyard millet (Echinochloa frumentacae) in type II diabetics. J. Food Sci. Technol. 2014, 51, 392-395. [CrossRef] [PubMed]

35. Marventano, S.; Vetrani, C.; Vitale, M.; Godos, J.; Riccardi, G.; Grosso, G. Whole Grain Intake and Glycaemic Control in Healthy Subjects: A Systematic Review and Meta-Analysis of Randomized Controlled Trials. Nutrients 2017, 9. [CrossRef] [PubMed]

36. Mozaffarian, D.; Hao, T.; Rimm, E.B.; Willettand, W.C.; Hu, F.B. Changes in Diet and Lifestyle and LongTerm Weight Gain in Women and Men. NEJM 2011, 364, 2392-2404. [CrossRef] [PubMed]

37. Hassapidou, M.; Fotiadou, E.; Maglara, E.; Papadopoulou, S.K. Energy Intake, Diet Composition, Energy Expenditure, and Body Fatness of Adolescents in Northern Greece. Obesity 2006, 14, 855-862. [CrossRef]

38. Halton, T.L.; Willett, W.C.; Liu, S.; Manson, J.E.; Stampfer, M.J.; Hu, F.B. Potato and french fry consumption and risk of type 2 diabetes in women. Am. J. Clin. Nutr. 2006, 83, 284-290. [CrossRef] [PubMed]

39. Noriko, M.M.; Nakamura, T.; Ohno, Y. New indicators for the evaluation of community policies based on period and cohort effects in cerebrovascular disease mortality rates. J. Jpn. Hosp. Assoc. 2009, 28, 79-85.

40. Shi, H.; Wang, J.; Wang, Q.; Liu, C. The association between food intolerance and eating habits among middle-aged population in north and south China. CMA 2013, 29, 263-267.

(C) 2019 by the authors. Licensee MDPI, Basel, Switzerland. This article is an open access article distributed under the terms and conditions of the Creative Commons Attribution (CC BY) license (http://creativecommons.org/licenses/by/4.0/). 